\title{
Anti-il-5 in pediatric allergic diseases
}

\author{
Laura Tenero ${ }^{1}$, Elisa Arturi ${ }^{2}$, Michele Piazza ${ }^{3}$, and Giorgio Piacentini ${ }^{2}$ \\ ${ }^{1}$ University of Verona \\ ${ }^{2}$ Università di Verona \\ ${ }^{3}$ Universita' di Verona, Clinica Pediatrica
}

August 28, 2020

\begin{abstract}
Interleukin (IL)-5 is a potent mediator of the inflammatory cascade in the allergic response.Its predominant role in atopic reactions makes this cytokine an ideal target for blocking the eosinophilic inflammatory hyper-responsiveness to allergens. The management of allergic diseases in childhood - such as severe asthma, atopic dermatitis, and eosinophilic esophagitis - is a challenge. In particular, there are concerns regarding the use of high-dose corticosteroids. Over the last few years, biologics targeting IL-5 or IL-5 receptor - that are mepolizumab, reslizumab, and benralizumab - represent a new, promising, and more personalized therapeutic option.
\end{abstract}

\section{Anti-il-5 in pediatric allergic diseases}

Laura Tenero, Elisa Arturi, Michele Piazza, Giorgio Piacentini

Affiliation : Paediatric section, Department of Surgery, Dentistry, Paediatrics, and Gynaecology, University of Verona, Verona, Italy.

Abstract :

Interleukin (IL)-5 is a potent mediator of the inflammatory cascade in the allergic response. Its predominant role in atopic reactions makes this cytokine an ideal target for blocking the eosinophilic inflammatory hyperresponsiveness to allergens.

The management of allergic diseases in childhood - such as severe asthma, atopic dermatitis, and eosinophilic esophagitis - is a challenge. In particular, there are concerns regarding the use of high-dose corticosteroids. Over the last few years, biologics targeting IL-5 or IL-5 receptor - that are mepolizumab, reslizumab, and benralizumab - represent a new, promising, and more personalized therapeutic option.

Key message : Biologics targeting IL-5 or IL-5 receptor may represent effective therapeutic options in children with severe allergies, once defined their benefit-risk profile in the pediatric age.

Keywords : IL-5, mepolizumab, reslizumab, benralizumab, asthma, children, biologics

\section{Corresponding Author :}

Giorgio Piacentini,

Paediatric Section,

Department of Surgery, Dentistry,

Paediatrics, and Gynaecology, University of

Verona, AOUI Verona, P.le Stefani 1, 37126 
Verona.

Email: giorgio.piacentini@univr.it

\section{Introduction}

Interleukin (IL)-5 is a potent mediator of the inflammatory cascade in the allergic response. It binds to the $\alpha$-chain of the IL-5 receptor (IL-5R) and modulates the eosinophil maturation in the bone marrow, recruitment, and activation at sites of allergic inflammation. Interleukin-5 also regulates the functions of basophils and mast cells, enhancing the release of their mediators.

Its predominant role in atopic reactions makes this cytokine an ideal target for blocking the eosinophilic inflammatory hyper-responsiveness to allergens.

The management of allergic diseases in childhood - such as severe asthma, atopic dermatitis, and eosinophilic esophagitis - is a challenge. In particular, there are concerns regarding the use of high-dose corticosteroids. Over the last few years, biologics targeting IL-5 or IL-5 receptor - that are mepolizumab, reslizumab, and benralizumab - represent a new, promising, and more personalized therapeutic option.

\section{Mepolizumab}

Mepolizumab neutralizes IL-5, through the inactivation of the signaling of IL-5 with the receptor and limiting the proliferation and activation of eosinophils. Mepolizumab is registered for use in adolescents ( $>12$ years) by the FDA and in children ( $>6$ years) by the EMA. It is indicated as an add-on maintenance therapy of severe asthma in patients with circulating eosinophils count $>150$ cells $/ \mu \mathrm{L}$, or $>300 \mathrm{cell} / \mu \mathrm{L}$ in the last year, unresponsive to standard treatment, with two or more severe exacerbations every year and/or dependency on systemic corticosteroids. ${ }^{1,2}$ Several studies confirm its effectiveness and tolerability in these patients. In contrast, no benefit is demonstrated in milder asthma that is not necessarily eosinophilic. Mepolizumab is administered subcutaneously every four weeks at a dose of $40 \mathrm{mg}$ for children, at $100 \mathrm{mg}$ for adolescents and adults. Adverse events include headaches, eczema, and nasal congestion. No case of anaphylaxis is reported. The response to the drug can be assessed at 12 months to determine whether to continue therapy. Long-term use could maintain a stable effect. However, the optimal duration of therapy is unclear. Mepolizumab reduces the number of eosinophils, improving the control of asthma symptoms, and decreasing the hospitalization rate. A Cochrane meta-analysis of mepolizumab vs. placebo in asthma, including 1707 patients aged 12 years and over, shows a reduction of exacerbation rates (of $53 \%$ with 95\% CI 37-65) and increase in FEV1 from baseline $(\mathrm{p}=0.03)$ for ones randomized to subcutaneous mepolizumab. Another study demonstrates a prednisone dose reduction 2.39 times greater with mepolizumab (95\% CI 1.25-4.56) compared to placebo. ${ }^{3}$.

The approval of mepolizumab in children (6-11 years) is based on the effectiveness data gained in the few trials enrolling children, and on the data about adolescents. ${ }^{4}$ Further researches are still ongoing.

For atopic dermatitis, mepolizumab shows only a modest improvement in clinical so far. Therefore, its application may be tried only in selected refractory patients. ${ }^{5}$

In the pediatric management of eosinophilic esophagitis, the role of mepolizumab is unclear. A multicenter study treats 59 children with different doses of mepolizumab: the decrease in esophageal eosinophil counts is shown, but there is a limited effect on clinical symptoms. ${ }^{6}$ Thus, the therapy is off-label and not clearly of benefit at this time.

\section{Reslizumab}

Reslizumab binds to IL-5, hindering it from stimulating the activation of eosinophils. Reslizumab, at present, is approved by FDA and EMA as add-on maintenance therapy of severe asthma only in adults with the eosinophilic phenotype (blood eosinophil count of $400 / \mathrm{microL}$ ) and at least three severe exacerbations in the last year, despite the standard treatment. The administration is intravenous $(3 \mathrm{mg} / \mathrm{kg})$ every four weeks, placed in a prepared setting for anaphylaxis (rate 0,3\%). Reslizumab leads to better control of asthma symptoms and improvement in lung function. According to two multicenter trials of Castro et al. on 953 
adolescents and adults with uncontrolled asthma, the reslizumab group experiences a significant reduction in exacerbations and a persistent improvement of FEV1 through 52 weeks. ${ }^{7}$ In contrast, other analyses in adolescents show a paradoxical increase in asthma exacerbations. To the present, reslizumab is not approved for children and adolescents.

Reslizumab is also evaluated as a treatment of eosinophilic esophagitis. In a randomized controlled trial involving 226 children and adolescents, the reslizumab group obtains a significant reduction in eosinophil counts compared with placebo. However, improvements in symptoms are observed in all treatment groups and are not associated with changes in oesophageal eosinophil counts. ${ }^{8}$ Opposite results are shown in smaller trials: long-term treatment with intravenous reslizumab exhibits no relapse or disease progression. Since its role is still uncertain, FDA does not approve reslizumab in children.

\section{Benralizumab}

Benralizumab is a monoclonal antibody direct against the $\alpha$-subunit of the IL- 5 receptor expressed on eosinophil and basophil, inhibiting the signaling and inducing cytotoxicity. Benralizumab is approved by EMA for adults and by FDA for adolescents with severe eosinophilic asthma. Benralizumab is given subcutaneously at a dose of $30 \mathrm{mg}$ every four weeks for the first three doses and then $30 \mathrm{mg}$ every eight weeks. Long-term efficacy over one year has been demonstrated. Frequent adverse events include headache and pharyngitis; anaphylaxis is rare. Benralizumab induces eosinophilic depletion in blood, sputum, and airway mucosa. In a trial of 1306 asthmatic adolescents and adults randomly assigned to benralizumab or placebo for 60 weeks, the annual exacerbation rate is reduced in the benralizumab group (RR 0.64, 95\% CI 0.490.85). ${ }^{9}$ Several studies - lasting up to years - show similar significant results. The glucocorticoid-sparing effect is examined in a 28 -week multicenter trial: the odds of reduction in the oral glucocorticoid dose with benralizumab are 4.09 times that with placebo. ${ }^{10}$

Busse and colleagues indirectly compared the efficacy of mepolizumab, benralizumab and reslizumab in patients aged 12 years or older with severe eosinophilic asthma, when grouped by patients' blood eosinophil counts, which are known to influence treatment effect. ${ }^{11}$ The study shows that mepolizumab reduces exacerbations by $34 \%-45 \%$ versus benralizumab across subgroups, and by $45 \%$ versus reslizumab in the [?]400cells/ $\mu \mathrm{L}$ subgroup. Moreover, mepolizumab is more effective in asthma control. There are no critical differences in lung function measured by change from baseline in pre-bronchodilator $\mathrm{FEV}_{1} \cdot{ }^{11}$

In summary, targeted therapies with anti-IL-5 or anti-IL-5R $\alpha$ approved in children include mepolizumab ( $>6$ years) and benralizumab (>12 years); reslizumab is today still off-label.

In case of severe refractory asthma, they offer a more personalized add-on treatment that results effective for reducing exacerbations and improves the asthma quality of life in the long-term.

For the management of eosinophilic esophagitis, the standard therapies such as diet elimination or corticosteroids can give concerns in some children, including lack of effectiveness, negative impact on the quality of life, or significant toxicity. Biologics can be a new strategy option, but more results collected specifically from pediatric clinical trials are needed.

In conclusion, anti-IL-5 agents are safe and effective in a short- and medium-term treatment of adult patients, whereas information for optimal therapy duration and long-term use is still lacking. Moreover, further researches are necessary for patients eligible for more than one treatment to find the adequate drug for each case through an evidence-based choice. ${ }^{12}$

Several studies about the efficacy and safety in pediatric patients are currently ongoing.

It's necessary to recruit large numbers of characterized children, with a wide range of endotypes with associated biomarkers prospectively measured, to predict the responders and compare the biologics.

\section{References}


1. Tenero L, Rossignoli S, Piacentini G. Severe asthma: When to resort to biological agents. Pediatr Allergy Immunol . 2020 febbraio; 31 Suppl 24: 37-39.

2. Licari A, Castagnoli R, Brambilla I, Marseglia A, Tosca MA, Marseglia GL, et al. Asthma Endotyping and Biomarkers in Childhood Asthma. Pediatr Allergy Immunol Pulmonol. 2018;31:44-55.

3. Farne HA, Wilson A, Powell C, Bax L, Milan SJ. Anti-IL5 therapies for asthma. Cochrane Database of Systematic Reviews 2017, Issue 9. Art. No.: CD010834.

4. Gupta A, Steinfeld J, Price R, Azmi J, Bradford E, Yancey S. Mepolizumab for severe eosinophilic asthma: a comparison of efficacy in children, adolescents, and adults. Eur Respir J. 2018. 52:PA5447.

5. Wollenberg A, Barbarot S, Bieber T, Christen-Zaech S, Deleuran M, Fink-Wagner A. Consensus based European guidelines for treatment of atopic eczema (atopic dermatitis) in adults and children: part II. J Eur Acad Dermatol Venereol 2018;32:850-878.

6. Assa'ad AH, Gupta SK, Collins MH, Thomson M, Heath AT, Smith DA, et al. An antibody against IL-5 reduces numbers of esophageal intraepithelial eosinophils in children with eosinophilic esophagitis. Gastroenterology. 2011;141:1593.

7. Castro M, Zangrilli J, Wechsler ME, Bateman ED, Brusselle GG, Bardin P, et al. Reslizumab for inadequately controlled asthma with elevated blood eosinophil counts: results from two multicentre, parallel, double-blind, randomised, placebo-controlled, phase 3 trials. Lancet Respir Med. 2015;3:355366 .

8. Spergel JM, Rothenberg ME, Collins MH, Furuta GT, Markowitz JE, Fuchs G 3rd, et al. Reslizumab in children and adolescents with eosinophilic esophagitis: results of a double-blind, randomized, placebocontrolled trial. J Allergy Clin Immunol. 2012;129:456-463, 463.e1-3.

9. FitzGerald JM, Bleecker ER, Nair P, Korn S, Ohta K, Lommatzsch M, et al. Benralizumab, an antiinterleukin-5 receptoramonoclonal antibody, as add-on treatment for patients with severe, uncontrolled, eosinophilic asthma (CALIMA): a randomised, double-blind, placebo-controlled phase 3 trial. Lancet. 2016;388:2128.

10. Nair P, Wenzel S, Rabe KF, Bourdin A, Lugogo NL, Kuna P, et al. Oral Glucocorticoid-Sparing Effect of Benralizumab in Severe Asthma. N Engl J Med. 2017;376:2448.

11. Busse W, Chupp G, Nagase H, Albers FC, Doyle S, Shen Q, et al. Anti-IL-5 treatments in patients with severe asthma by blood eosinophil thresholds: Indirect treatment comparison. J Allergy Clin Immunol. 2019;143:190-200.e20.

12. Licari A, Manti S, Castagnoli R, Parisi GF, Salpietro C, Leonardi S, et al. Targeted Therapy for Severe Asthma in Children and Adolescents: Current and Future Perspectives. Paediatr Drugs. 2019;21:215237. 